\title{
Beyond loot boxes: A variety of gambling-like practices in video games are linked to both problem gambling and disordered gaming
}

\author{
David Zendle ${ }^{\text {Corresp. } 1}$ \\ ${ }^{1}$ Computer Science, University of York, York, United Kingdom \\ Corresponding Author: David Zendle \\ Email address: david.zendle@york.ac.uk
}

A variety of practices have recently emerged which relate to both video games and gambling. These range from opening loot boxes, to esports betting, real-money video gaming, token wagering, and social casino spending. It is unknown either how harmful or how widespread many of these activities are. A sample of 1081 adults from the UK aged $18+$ was therefore recruited. This sample was purposively recruited via quota sampling to represent the UK population in terms of sex, age, and ethnicity. Engagement in all forms of gaming-related practices were significantly associated with both problem gambling and disordered gaming. $18.5 \%$ of the sample had engaged in these activities at least once in the new year. These results suggest a convergent ecosystem of practices that relate to both video games and gambling. Engagement in each of these activities is linked to problem gambling. However, it remains unclear whether engagement in these activities causes problem gambling. 


\section{Beyond loot boxes: There are a variety of forms of}

2 gambling and gambling-like behaviour that relate to

3 video games, and they are all linked to both problem

4 gambling and disordered gaming

David Zendle ${ }^{1}$

${ }^{1}$ Department of Computer Science, University of York, York, United Kingdom

Corresponding Author:

David Zendle

Department of Computer Science, University of York, York, YO10 5GH, United Kingdom

Email address: David.zendle@york.ac.uk

\section{Abstract}

A variety of practices have recently emerged which relate to both video games and gambling.

These range from opening loot boxes, to esports betting, real-money video gaming, token wagering, and social casino spending. It is unknown either how harmful or how widespread many of these activities are.

A sample of 1081 adults from the UK aged $18+$ was therefore recruited. This sample was purposively recruited via quota sampling to represent the UK population in terms of sex, age, and ethnicity. Engagement in all forms of gaming-related practices were significantly associated with both problem gambling and disordered gaming. $18.5 \%$ of the sample had engaged in these activities at least once in the new year.

These results suggest a convergent ecosystem of practices that relate to both video games and gambling. Engagement in each of these activities is linked to problem gambling. However, it remains unclear whether engagement in these activities causes problem gambling.

\section{Introduction}

A blurring of the lines has occurred between video games and gambling activities. The most widely-discussed example of this convergence are loot boxes: Items in video games that may be bought for real-world money, but which contain randomized contents (Zendle et al., 2020).

Loot boxes share several formal features with gambling, and there has been widespread interest in the idea that engaging with loot boxes may lead to problem gambling (Brooks \& Clark, 2019; Drummond \& Sauer, 2018; King \& Delfabbro, 2018, 2020; Li et al., 2019). Indeed, engagement with loot boxes has been repeatedly linked to problem gambling: The more that 
41 gamers use loot boxes, the more severe their gambling problems tend to be (Brooks \& Clark, 42 2019; Li et al., 2019; Zendle, 2019a; Zendle et al., 2018, 2019; Zendle \& Cairns, 2018, 2019). It

43 is important to note that the robustness of this link does not necessarily mean that loot box

44 spending causes problem gambling: It may well be the case that these factors are instead linked 45 because problem gamblers are more likely to engage with loot boxes, or that some third factor 46 (such as impulsivity) drives engagement with both loot boxes and gambling activities.

47

48

49

50

51

52

53

54

55

56

57

58

59

60

61

62

63

64

65

66

67

68

69

70

71

72

73

74

75

76

77

78

79

80

81

82

83
Problem gambling refers to a pattern of gambling engagement that is so extreme it causes an individual to have important problems in various aspects of their life (Raylu \& Oei, 2004). It has been linked to depression, anxiety, bankruptcy, and suicidality (Barrault \& Varescon, 2013; Grant et al., 2010; Petry \& Kiluk, 2002). It is commonly considered a serious public health issue, and the similarities and associations between loot boxes and gambling have been sufficient to lead to global regulatory interest in loot boxes. For example, in the United Kingdom, similarities regarding loot boxes prompted a parliamentary inquiry into their effects (Kent, 2019). Evidence of their relationship to gambling was sufficient to prompt the UK government to announce a planned holistic reform of gambling law in order to address issues related to loot boxes(Hymas, 2019).

However, loot boxes are not alone in blurring lines between gambling and video games. A variety of gambling and gambling-like practices have recently emerged in the video game domain. These range from betting on esports, to spending money on social casino games, to more obscure practices like token wagering and real-money video gaming.

As noted above, there are a variety of different practices that constitute an intersection of gambling and video games. Given the interest that has been shown to the intersection of gambling and video games that loot boxes constitute, one might imagine that a rich literature exists to explore questions of prevalence and potential harm when it comes to these activities. However, little research has attempted to estimate either how common several of these practices are, or whether they share a link to problem gambling in a similar fashion to loot boxes.

Researchers have proposed that loot boxes share so many formal similarities with gambling that they may act as a gateway to engagement with gambling amongst gamers, and hence the development of problem gambling (Drummond \& Sauer, 2018). Similarly, researchers have proposed that loot box spending may be linked to disordered gaming. Disordered gaming refers to a possible condition in which persistent and recurrent engagement with video games leads to significant impairment or distress (American Psychiatric Association, 2013). Such a relationship may lead to a problematic situation in which vulnerable individuals who play games to an excessive degree may be exposed to other potential harms (Li et al., 2019).

Similar concerns may be raised about other gambling and gambling-like practices that are associated with video games. An overview of each of these relevant practices is given below, as is an overview of research into engagement with loot boxes.

Peer] reviewing PDF | (2020:01:45265:3:0:NEW 30 May 2020) 


\section{Esports betting}

85 Esports betting refers to the practice of placing wagers on the outcomes of multiplayer videogame competitions. Esports spectatorship has become increasingly popular in recent years. Indeed, the final of the 2018 League of Legends World Championships attracted over 44 million concurrent viewers (Goslin, 2018). This has led to a rapidly-growing betting culture, with mainstream betting providers commonly hosting esports streams alongside traditional sports like rugby and football. One industry report estimates that $\$ 5.5$ billion was bet on esports in 2016 alone, with this figure set to more than double to $\$ 12.9$ billion by 2020 (C. Smith, 2019).

Research has suggested that esports betting may be linked to esports spectatorship and video gaming, and that esports betting may be linked more strongly to problem gambling than traditional sports betting (Gainsbury et al., 2017; Macey \& Hamari, 2018). However, the overall prevalence of esports betting is unknown.

\section{Social casino games}

98

99

100

101

102

103

104

105

106

107

108

109

110

111

112

113

114

\section{5}

116

117

118

119

120

In social casino games, players can spend real money to engage in simulated games of chance such as roulette or slot machine play. These games are commonly presented and sold in mobile phone app stores as a form of video game. They differ from conventional gambling in that players cannot win real-money rewards for their spending (M. Gainsbury et al., 2014). There is evidence that users of social casino games may migrate to engagement in conventional gambling activities (Gainsbury et al., 2016). Some social casino providers claim to have millions, or tens of millions, of active players (Kim et al., 2015). The prevalence of social casino spending may therefore be high. A recent large-scale survey of Canadian adolescents $(n=10,035)$ found that as many as $12.4 \%$ of respondents had recently played social casino games (Veselka et al., 2018). However, the generalisability of this figure to other populations is unclear.

\section{Real-money video gaming}

Real-money video games integrate the ability for gamers to wager real money on the outcomes of their in-game efforts. For example, in Strike! eSports Bowling, players are prompted in-game to wager money on their success at games of online ten-pin bowling. Video games incorporating this mechanic range from sports games to Tetris-like puzzles such as Block Blitz (Zendle, $2019 b)$. The prevalence of engagement in these activities and links to engagement with gambling are unclear.

\section{Token wagering}

In games that incorporate token wagering, players do not wager real-world money on the outcome of their in-game activities. Instead, they engage in the related practice of wagering tokens or points. The rewards from winning wagers can then be redeemed for in-game rewards. This form of wagering is common in popular games such as DOTA 2, which is estimated to have over 11 million unique players each month (Dota 2 Crosses 11 Million Players, 2019; Dota 
121

122

123

124

125

126

127

128

129

130

131

132

133

134

135

136

137

138

139

140

141

142

143

144

145

146

147

148

149

150

151

152

153

154

155

156

157

158

159

2 - The International Battle Pass 2019, n.d.). After conducting a literature search, we have failed to find a single academic source which examines either the potential effects or the prevalence of token wagering.

\section{Loot boxes}

Loot boxes are items in video games that are bought with real-world money but contain randomised contents whose value is uncertain at the point of purchase. For example, players of the first-person shooter game Counter-Strike: Global Offensive can spend money to purchase sealed 'weapon cases'. The contents of these cases may be rare and valuable, or they may be common and worthless. Players are not aware of the value of loot box contents when they make their purchase. Because of formal similarities between loot boxes and gambling, there are concerns that they may provide a gateway to gambling amongst gamers (Drummond \& Sauer, 2018). Research has repeatedly linked loot box spending to problem gambling, with effect sizes in excess of $\eta^{2}=0.04$ repeatedly observed in the literature (Brooks \& Clark, 2019; Zendle et al., 2019; Zendle \& Cairns, 2018, 2019). In media effects research, it is common to refer to effects in excess of this cut-off as potentially 'clinically significant' in nature, as effects smaller than this may be of insufficient magnitude to warrant a clinician's attention (Ferguson, 2009). However, the temporal order of these links is unclear: They may represent a case in which loot boxes act as a gateway to problem gambling; alternatively, they may represent a case in which preexisting gambling problems causes gamers to spend more money on loot boxes (Zendle et al., 2019). Indeed, they may represent a situation in which a third factor is responsible for both behaviours: For example, those who have access to loot boxes may also tend to have access to similar technology-driven forms of gambling such as internet casinos and bingo.

One may also speculate that the financial cost associated with loot boxes may be so low as to not be potentially harmful. Indeed, recent surveys have shown relatively low mean spending on loot boxes amongst gamers (Drummond et al., 2020; Zendle \& Cairns, 2018). However, the range of spending on loot boxes in video games is far from certain. The analyses highlighted above were conducted over samples of limited representativeness: Zendle and Cairns, for example, recruited participants from reddit, a popular online bulletin board. Using their results to estimate mean spend may be of limited utility.

Loot box spending has also been linked to disordered gaming, suggesting that it may be more common amongst vulnerable gamers (Li et al., 2019). No studies have thus far examined what proportion of the population engage in this behaviour. The prevalence of loot box spending is therefore unclear.

\section{Watching gambling and loot box spending online}

Live game streaming services like Twitch and video sharing websites like Youtube allow individuals to broadcast both live and pre-recorded videos online. These platforms are also commonly used to broadcast live videos of individuals engaging in both gambling activities, and 
160

161

162

163

164

165

166

167

168

169

170

171

172

173

174

175

176

\section{7}

178

179

180

181

182

183

184

185

186

187

188

189

190

191

192

193

194

195

196

197

opening loot boxes (Kent, 2018; Klepek, 2019). The prevalence of watching such streams, and the links between this kind of engagement with both video game play and gambling is unclear.

\section{Summary}

The lines between video games and gambling have been thoroughly blurred. A variety of practices exist that incorporate elements of both video games and gambling: esports betting, social casino spending, real-money gaming, token wagering, loot box spending, and the watching of gambling and loot box opening videos online. Excepting loot box spending, both the prevalence of these practices and their links with potentially important factors such as problem gambling and disordered gaming are currently unclear. The objective of this study is to begin investigating these issues.

In the present research, we therefore measure engagement in the variety of gambling-related video game practices outlined above. We then estimate both the prevalence of these practices; and we measure the relationship between each of these practices and both problem gambling and disordered gaming.

\section{Materials \& Methods}

\section{Design}

We conducted an online survey with a sample of adults aged 18+ from the United Kingdom. This sample was recruited for us by Prolific Academic, and was quota-sampled to be nationally representative in terms of ethnic, sex, and age subgroups as calculated by the 2011 UK Census. Data were collected remotely via Prolific, and researchers did not have control over who was selected for inclusion within the sample, reducing the potential for selection bias.

Prolific Academic are an online panel provider. The participant pool for Prolific Academic consists of a group of individuals who have previously signed up with this service provider in order to take part in experiments and other studies. These participants may check their Prolific accounts at any point that is convenient to them. These accounts will then list studies that are available for them to take part in. After participation, individuals are remunerated for their efforts. In cases such as this, recruitment takes the form of offering relevant individuals (i.e. those that match the quota sampling frame described in this document) the opportunity to take part in this study.

Interestingly, this format means that traditional response rates cannot be obtained in this context. In a traditional survey (such as a 'mail shot'), researchers might physically post out 1,000 envelopes to participants. If they received 900 responses from this initial invitation, they would be able to quantify a response rate of $90 \%$. There is no easily-accessible analogue in the context of online providers like Prolific and Amazon Mechanical Turk. If, for example, an 
198 Amazon Mechanical Turk study is available to a million mTurkers and is filled in by a sample of 1,000 , does this correspond to a participation rate of $0.1 \%$ ? We would argue that it does not.

200

201

202

203

204

205

206

207

208

209

210

211

212

213

214

215

216

217

218

219

220

221

222

223

224

225

226

\section{Measurements}

The frequency of participants engagement with gambling activities were measured by asking participants how frequently they had engaged in a variety of behaviours during the past 12 months. Answers to these questions were measured on an 8-point scale: (1) I have never done this; (2) Not at all in the past 12 months, but I have done this before then; (3) Less than 10 times in total; (4) Once a month; (5) 2-3 times a month; (6) Once a week; (7) 2-3 times a week; (8) 4 or more times a week;. 11 traditional forms of gambling were measured. These were:

1. Purchasing lottery tickets

2. Purchasing instant win / scratch cards

3. Betting on sports events (excluding esports)

4. Betting on horse or dog racing

5. Playing bingo for money in person

6. Playing bingo for money online

7. Playing games of skill for money against other individuals

8. Playing slot machines in person

9. Playing slot machines online

10. Playing casino table games in a casino

11. Playing casino table games online

In addition to this, gaming-related forms of gambling and gambling-like behaviour were measured via the same procedure. The forms of gaming-related behaviour that were measured were:

1. Esports betting ("Esports betting is the practice of wagering real money on the outcome of video game competitions or matches. Over the past 12 months, how often would you say that you have engaged in esports betting?")

2. Loot box spending (Question given below)

3. Social casino spending ("Social casino games refer to apps in which players can play simulated gambling games. Examples of such games are Zynga Poker and Pop! Slots. 
These games differ from traditional gambling because whilst players may pay real-world money to play these games, they cannot receive monetary rewards from them. Over the past 12 months, how often would you say that you have spent money in social casino games?")

4. Real-money video gaming ("Real-money video games are video games where you can wager real money on your in-game success, in the hopes of winning more real money. Examples of such games include Strike! Bowling, and Pro Pool. Over the past 12 months, how often would you say that you have spent money on real-money video games?")

5. Token wagering ("Token wagering is a term that is used to refer to the practice of wagering tokens or points on the outcome of video game competitions or matches. Over the past 12 months, how often would you say that you have engaged in token wagering?")

6. Watching loot box openings online (live)

7. Watching loot box openings (pre-recorded)

8. Watching gambling online (live) ("Some people watch others gamble live via video streaming service or websites like Twitch. Over the past 12 months, how often would you say that you have engaged in watching others gamble live via a video streaming service or website?")

9. Watching gambling online (pre-recorded) ("Some people watch others gamble via prerecorded clips on websites like Youtube. Over the past 12 months, how often would you say that you have engaged in watching others gamble via a pre-recorded clip on a website?)

In order to measure loot box spending, a more time-consuming procedure was employed. 
278

279

280

281

282

283

284

285

286

287

288

289

290

291

292

293

294

295

296

"'Loot boxes' refer to any items or rewards in a video game that are paid for with real money, but contain randomised contents. Not all loot boxes literally look like boxes. For example:

- Players of Counter-Strike: Global Offensive can pay real-world money to open 'weapon cases' that contain a random skin for their in-game gun. Some skins are more rare than others.

- Players of FIFA Ultimate Team can pay real-world money to buy 'player packs' of new footballers. The contents of player packs are randomised, and when paying their money, gamers don't know if they're paying for good players or poor players.

- Players of Fire Emblem: Heroes can pay real-world money for the chance to obtain a random in-game hero."

They were then asked "Given the definition of loot boxes above, have you played a game during the last 12 months where you had the opportunity to buy a loot box?" and "Over the past 12 months, how often would you say that you have purchased loot boxes?"

It is important to note that in order to ensure that participants gave accurate responses, each of these novel forms of gaming-related behaviour was introduced to participants via a short piece of text prior to asking participants to endorse an answer. For example, esports betting was introduced with the text "Esports betting is the practice of wagering real money on the outcome of video game competitions or matches."; watching gambling online (live) was introduced with the text "Some people watch others gamble live via video streaming service or websites like Twitch". The exact questions asked for each item surveyed are available at the OSF repository listed in the data accessibility statement for this manuscript.

\section{Two aggregate measures of both engagement in any traditional form of gambling and} engagement in any form of video game and gambling-related activity were formed by taking a participant's maximum response to either any of the traditional gambling frequency measures, or any of the video game-related frequency measures. In order to be as parsimonious as possible with estimating the frequency of video game-related gambling and gambling-like behaviour, both watching gambling live and on a pre-recorded basis were excluded from this calculation.

Problem gambling severity was measured through administration of the problem gambling severity index (PGSI). This 9-item scale is commonly used to measure problem gambling, and has been extensively validated (Ferris \& Wynne, 2001; Orford et al., 2010; Turner et al., 2018). Each question in the PGSI measures how frequently an individual has engaged in a problematic gambling-related behaviour during the past year (e.g. "Thinking about the last 12 months, how often have you bet more than you could really afford to lose?"). Answers are given on a fourpoint scale ranging from "Never" to "Almost always", and scores are summed to create an overall index of problem gambling severity ranging from 0 to 27 . In this instance, diagnostic categories are formed according to (Currie et al., 2013): Individuals who score 0 on the PGSI are categorised as 'non problem gamblers'; 1-4 as 'low risk gamblers'; 5-7 as 'moderate risk 
297

298

299

300

301

302

303

304

305

306

307

308

309

310

311

312

313

314

315

316

317

318

319

320

321

322

323

324

325

326

327

328

329

330

331

332

333

334

335

336

337

338

gamblers' and 8+ as 'problem gamblers'. Other scoring schemes for the PGSI calculate low risk gamblers and moderate gamblers differently; there is some evidence that this scheme yields greater validity (Currie et al., 2013). The Cronbach's Alpha for this scale was calculated in this instance as 0.85 .

Disordered gaming was measured via administration of the Internet gaming disorder scale (Lemmens et al., 2015). This 9-item instrument presents a series of Yes/No questions regarding the activities that individuals have engaged in during the past year that map to APA (American Psychiatric Association) criteria for the presence of gaming disorder (e.g. "Have you had arguments with others about the consequences of your gaming behaviour?"). Endorsement of 5 or more criteria is used to screen individuals as positive for the presence of disordered gaming. This is based on diagnostic criteria for Internet Gaming Disorder found in the 5th edition of the Diagnostic and Statistical Manual for Mental Disorders. The KR-20 for this scale was calculated in this instance as 0.79 .

Ethical approval for this research was granted by the York St John University Cross-

Departmental Ethics Board, under submission code 2159. It should be noted that the lead author's current affiliation is to the University of York, and not York St. John University. The reason for the difference in the IRB and the current author's host institution is that this study was conducted during the author's transition from a lectureship at York St. John University to a lectureship at the University of York.

Informed consent was gathered from each participant. As this was an online study, this took the form of a brief description of the kinds of data that would be collected and how it would be used, and an opportunity to opt in to the study by ticking a box. The full script for this study (including the specific presentation of this informed consent procedure) is available at the OSF repository detailed below.

\section{Participants}

Overall, an initial sample of 1201 individuals was collected between August $30^{\text {th }} 2019$ and September $9^{\text {th }}$ 2019. Participants were recruited via a deliberately ambiguous study descriptor that was designed to minimize any potential for self-selection bias. This message read as follows: "In this study you will be asked to provide some demographic details, and then some information about activities that you engage in.". In addition to the 1201 participants whose data was collected, a further 21 individuals began, but then returned the study.

Participants were reimbursed $£ 0.70$ for taking part in the study. On average, participants took less than 8 minutes to complete and submit their responses to the study, with an average reimbursement rate of $£ 5.65 / \mathrm{hr}$.

After data were recorded, participants were then excluded from the sample on the basis of either (a) failing an initial seriousness check or (b) failing either of two later attention checks.

Peer] reviewing PDF | (2020:01:45265:3:0:NEW 30 May 2020) 
First, participants were excluded on the basis of their response to a seriousness check. This

342 took the form of a series of four questions asking participants whether they had played specific

343 games. Two games listed ('Game of Glory' and 'Rise of Warriors') were fictitious. Any

344

345 participants who indicated that they had played these games were removed from the sample. 39 participants endorsed these items and were removed from the sample.

The attention check took the form of two separate questions which asked participants to give pre-specified answers in order to establish that they were paying attention to the survey. The first attention check question read "In order to check the reliability of your responses, please select 'Once a month' as the answer to this question". The second attention check question read "Please select 'No' as the answer to this question". 80 participants failed the first attention check, and a further 1 participant failed the second. These participants were removed from the study, leaving a total of 1081 participants.

353

354

355

Overall, 526 participants listed their gender as male, and 549 listed their gender as female. 6 gave other responses.

356

357

Overall, 926 participants listed their ethnicity as White; 29 as Mixed; 38 as Black; 76 as Asian, and 12 as Other.

359

360

Overall, 190 participants were aged 18-27, 176 participants were aged 28-37, 203 participants

361

362

363 were aged 38-47, 184 participants were aged 48-57, and 328 participants were aged 58+.

Overall, 24 of the 1081 participants (2.2\%) were classified as problem gamblers. A further 35 $(3.2 \%)$ were classified as moderate risk and $235(21.7 \%)$ as low risk. 79 of the 1081 participants

365

366 $(7.3 \%)$ were classified as disordered gamers.

368

369

370

371

372

373

374

375

376

377

378

379

380

381

382

Overall, 13 of the 526 male participants (2.4\%) were classified as problem gamblers, and 11 of the 549 female participants $(2.0 \%)$. None of the 6 participants giving other responses when asked about their gender were problem gamblers. 49 of the 526 male participants $(9.3 \%)$ were classified as disordered gamers, and 30 of the 549 female participants (5.4\%). None of the participants who gave other gender responses were classified as disordered gamers.

72 individuals had both engaged in traditional gambling and loot box spending in the past year. The majority of these (42) stated that they had engaged in traditional gambling prior to loot box spending; 17 had engaged in loot box spending prior to traditional gambling; and 13 responded 'Not sure / Not applicable' when asked.

35 individuals had both engaged in traditional gambling and social casino spending in the past year. The majority of these (23) stated that they had engaged in traditional gambling prior to social casino spending; 4 had engaged in social casino spending prior to traditional gambling; and 8 responded 'Not sure / Not applicable' when asked. 
38331 individuals had both engaged in traditional gambling and esports gambling in the past year.

384 The majority of these (23) stated that they had engaged in traditional gambling prior to esports 385 gambling; 4 had engaged in esports gambling prior to traditional gambling; and 4 responded 386 'Not sure / Not applicable' when asked.

387

388

389

390

391

392

393

394

395

396

397

398

399

400

401

402

403

404

405

406

407

408

409

410

411

412

413

414

415

416

417

418

419

420

421

422

423
20 individuals had both engaged in traditional gambling and token wagering in the past year. The majority of these (12) stated that they had engaged in traditional gambling prior to token wagering; 2 had engaged in token wagering prior to traditional gambling; and 6 responded 'Not sure / Not applicable' when asked.

\section{Results}

The proportion of the sample engaging in each of the traditional gambling and gaming-related gambling behaviours within the sample is shown below as Table $1.95 \%$ confidence intervals were calculated via the adjusted Wald procedure.

Spearman rank correlation coefficients were calculated for the relationship between ordinal responses (1-8) to questions regarding the frequency of engagement with each form of gamerelated practice and both problem gambling and disordered gaming. The result of this analysis is presented below as Table 2. In all relevant instances, continuous scores were calculated from the IGDS by counting the presence of a symptom as ' 1 ' and the absence of a symptom as ' 0 ', forming a numeric score ranging from 0 to 9 .

Spearman rank correlation coefficients were calculated for the relationship between ordinal responses (1-8) to questions regarding the frequency of engagement with each traditional form of gambling and each form of game-related practice. The results of this are given below as Table 3.

Spearman rank correlation coefficients were calculated for the relationship between ordinal responses (1-8) to questions regarding the frequency of engagement with each video-game related gambling practice and each other game-related practice. The results of this are given below as Table 4 . 
424

425

426

427

428

429

430

431

432

433

434

435

436

437

438

439

440

441

442

443

444

445

446

447

448

449

450

451

452

453

454

455

456

457

458

459

460

461

462

463

464

465

466

467

Exploratory Analyses

During peer review, a number of additional analyses were suggested by reviewers. We agreed with these reviewers that these analyses constituted an interesting exploration of the data, and report them below.

To begin with, it was suggested that we scrutinize our data for evidence of common method bias. Common method bias occurs when shared variance amongst variables is attributable to a common method used to measure these items. Lengthy and demanding procedures are thought to lead to common method bias, as individuals attempt to optimize their progress through a study by using a method other than valid responding (MacKenzie \& Podsakoff, 2012). The simplicity and short length of this study (less than 10 minutes mean completion time) may mitigate the risk of common method bias in our results. However, in order to address this issue, Harman's Single Factor Test was applied to our data. This is a common test for common method bias (Podsakoff et al., 2003). It involves entering all measured variables into an exploratory factor analysis in order to determine how much variance between items is attributable to a single unifying factor. A measurement above $50 \%$ is taken as evidence that common method bias may be present. However, in this case, a single factor was only able to explain $23.44 \%$ of variance. The limitations of this approach are described in our discussion.

Next, reviewers requested that analyses investigating the relationship between the novel activities under investigation and both problem gambling and disordered gaming were conducted that statistically took age and gender into account. In order to binarise gender, we removed the 6 members of the cohort who identified as neither male nor female before analyzing the data.

In many studies that address the predictors of problem gambling, such an analysis would be conducted via multiple linear regression. However, inspection of a plot of the residuals of an initial analysis revealed that the strongly non-normal nature of our outcome variable meant that any models build on this data would violate assumptions of multivariate normality. In order to address this, data was analysed via logistic regression-based approaches, which do not require this assumption.

First, the ability of age, gender, and gaming-related behaviours to predict problem gambling were analysed via a series of ordinal logistic regressions. Each regression had problem gambling (non-problematic, low-risk, moderate-risk, problem gambler) as predictor. Each analysis had gender, age, and the frequency of a single form of gaming-related behavior as outcomes. Assumptions of multicollinearity were addressed via the calculation of VIFs for each regression; in each case, all VIFs were below 4, indicating a lack of multicollinearity.

Assumptions of proportional odds were tested via the calculation of Brant's test for each ordinal logistic regression: In all cases, omnibus test statistics were non-significant $(p>0.05)$, indicating a failure to reject the null hypothesis that the relationship between each pair of outcome groups is the same. All p-values for all predictors across all analyses were also non-significant, with the exception of token wagering $(p=0.02)$. For this reason, analyses involving token wagering are

Peer) reviewing PDF | (2020:01:45265:3:0:NEW 30 May 2020) 
468

469

470

471

472

473

474

475

476

477

478

479

480

481

482

483

484

485

486

487

488

489

490

491

492

493

494

495

496

497

498

499

500

501

502

503

504

505

506

507

508

509

510

511

not reported below. Results of regressions involving problem gambling as outcome are depicted below as Table 5 .

First, the ability of age, gender, and gaming-related behaviours to predict disordered gaming were analysed via a series of logistic regressions. Each regression had disordered gaming (non-disordered, disordered) as predictor. Each analysis had gender, age, and a single form of gaming-related behavior as outcomes. Assumptions of multicollinearity were addressed via the calculation of VIFs for each regression; in each case, all VIFs were below 4, indicating a lack of multicollinearity. Results of logistic regression are depicted below as Table 6 .

It was suggested that the relationship between disordered gaming and problem gambling was calculated. Spearman's rho for these scores was calculated at $0.26(p<0.001)$, indicating a small to moderate relationship between these variables (equivalent $r^{2}=0.06$ ).

We then calculated the proportion of individuals who played games with loot boxes that spent money on them. Overall, 283 of the 1081 individuals in our sample had played a game with a loot box in it $(26.1 \%)$. Of these 283 , the majority $(n=160)$ had spent money on them $(56.5 \%)$.

Further exploratory analyses were then suggested and conducted on measurements regarding the precedence of gaming-related behaviours and traditional gambling. A subset of individuals in the study indicated that they had both engaged in some form of traditional gambling, and also indicated that they engaged in one of several forms of gaming-related behavior (esports betting, loot box spending, real money gaming, social casino spending, token wagering). These individuals were given a follow-up question for each practice, asking them which one they thought came first.

Finally, one may suggest that individuals may interpret items within the PGSI as referring to gaming-related activities rather than traditional gambling, leading to correlations between engagement in these activities and problem gambling. For example, one question asks individuals whether they have bet more than they could afford in the past 12 months. One might imagine an individual who thinks that they have 'bet more than they could afford' on loot boxes, and hence rates this question highly. In order to address whether this might occur, we counted how many individuals within our sample had a rating greater than zero on the PGSI despite not engaging in any traditional form of gambling. This was a rare occurrence: It was only the case for 6 participants.

\section{Discussion}

This study suggests that game-related gambling and gambling-like behaviours may be relatively widespread amongst UK adults. It also provides initial evidence that all of these practices are significantly linked to both problem gambling and disordered gaming.

Overall, a significant proportion of the sample had engaged in some form of gaming-related gambling or gambling-like practice $(18.5 \%)$. Within our sample, many of these practices appeared as widespread as some traditional forms of gambling. For example, engagement in

Peer) reviewing PDF | (2020:01:45265:3:0:NEW 30 May 2020) 
512 game-related practices was of comparable popularity to engagement in well-established forms 513 of traditional gambling such as playing slot machines online (14.4\%) or betting on horse or dog 514 racing $(21.7 \%)$. Indeed, just engaging in loot box spending $(7.8 \%)$ was of similar popularity to

515 engaging in established gambling activities such as playing games of skill for money (6.8\%) and 516 playing casino games in a casino (7.4\%). However, it is also important to note that whilst the 517 popularity of these specific gaming-related practices may be comparable with engagement in 518 specific forms of gambling, overall engagement with any form of traditional gambling within our 519 sample was much higher: $71.3 \%$ of the sample stated that they had engaged in some form of 520 traditional gambling in the past year.

521

522

523

524

525

526

527

It is interesting to note that this overall summary statistic tallies very closely with the most estimates published in the most recent British Gambling Prevalence Survey (BGPS) (Wardle et al., 2011). In the BGPS, $73 \%$ of adults aged $16+$ had engaged in gambling in the previous year: here, we see an overall engagement rate of $71.3 \%$. However, it is also key to point out that the rates obtained here also diverge in places from the measurement of prevalence in the BGPS. For example, scratch card use in the BGPS is estimated at $24 \%$ of the population. By contrast, $34.7 \%$ of participants in our study had purchased scratch cards in the past year. Most strikingly,

529

530 two percent of participants in the BGPS had engaged in playing online slots in the past year However, $14.4 \%$ of our sample stated that they had done this. The reason for this discrepancy is unclear: It seems likely that specific practices may have changed in popularity in the decade

532

533 separating our sampling from the most recent British Gambling Prevalence Survey. For example, online play may logically have increased in popularity during this period. However, it may be the case that the sample that we obtained overestimates the true national prevalence of

535

536

537 specific gambling activities.

538

Engagement with video-game related gambling practices in general was significantly linked to problem gambling (rho=0.23). Indeed, every single form of game-related gambling and

539

540

541

542 One might assume that the form of video-game related gambling practice that would be most

543 strongly linked to problem gambling was engagement in esports betting, as engagement in

544

545

546

547 gambling-like practice was significantly linked to problem gambling, including previously unstudied practices like token wagering and real-money video gaming. esports betting literally constitutes gambling and may therefore directly contribute to problem gambling severity.

548 However, whilst there was an important link between esports betting and problem gambling, there was an equally strong link between social casino spending and problem gambling (both

550 rho $=0.21$ ). Furthermore, links between problem gambling and watching gambling live on game

551 streaming services like Twitch was of comparable magnitude (rho=0.20). All of these links are of clinically-significant size. The phrase 'clinically significant' in this context refers to a benchmark

552

553 cut-off of $r=0.2$ that is commonly used in media effects research to indicate an effect that may be of practical importance to clinicians(Ferguson, 2009).

554 
555 The reason for these links is unclear: It may well be the case, for example, that watching others 556 gamble live might prompt an individual to gamble themselves, and therefore lead to the

557

558

559

560

561

562

563

564

565

566

567

568

569

570

571

572

573

574

575

576

577

578

579

580

581

582

583

584

585

586

587

588

589

590

591

592

593

594

595

596

597

598 development of problem gambling. Similarly, one might propose that engagement with simulated games of chance in a social casino context leads to individuals engaging in gambling, and hence the development of gambling problems. However, the direction of any causal link between these activities is unclear and should be the subject for further research. This is of particular importance when it comes to the live viewing of gambling activities on video game streaming services, which has thus far not been explored widely in the literature.

A significant relationship was observed here between the frequency with which an individual engages in loot box spending and their problem gambling. However, interestingly, the relationship seen here was smaller in size than effects observed previously. Previous work has observed a link between the amount of money that an individual spends on loot boxes in a single month and their problem gambling severity of approximately $\eta^{2}=0.05$ to 0.12 (Zendle et al., 2019; Zendle \& Cairns, 2018). The link between the frequency of engagement in loot box spending and problem gambling observed here was smaller: rho=0.14, equivalent to $\eta^{2}=0.02$. The reason for this difference in magnitude is unclear. It may be the case that previous studies have overestimated the size of this link; it may be that the way that engagement with loot box spending was measured here has conversely led to an underestimation of effect sizes in this instance. Further work is necessary to determine which of these is the case.

A potentially important relationship was observed between disordered gaming and game-related gambling and gambling-like behaviours. Every single form of game-related practice was linked to disordered gaming. Indeed, overall engagement in these behaviours was strongly linked to disordered gaming ( $r h o=0.43$ ). In other words, over $18 \%$ of the variance in gaming-related practices within the sample could be accounted for by the existence of a disordered relationship with video gaming. The specific practices that displayed the strongest links with disordered gaming all related to loot boxes: loot box spending (rho=0.41), watching loot box openings live online (rho=0.32), and watching pre-recorded loot box openings (rho=0.35). This echoes previous research which has established a link between loot boxes and disordered gaming ( $\mathrm{Li}$ et al., 2019). However, again, the specific reason for this link is unclear, and further qualitative and experimental work is necessary to determine precisely both why disordered gamers might disproportionately engage with loot boxes, and what the consequences of this engagement might be.

The more that individuals engaged in gaming-related gambling and gambling-like practices, the more likely they were to also engage in traditional gambling practices (rho=0.13). gamingrelated practices were particularly strongly linked to computer-mediated forms of gambling such as online slot machines (rho=0.27), and online casino games (rho=0.30). This is logical, considering all these activities necessarily involve using a computer or mobile device. There were also significant links between all forms of video-game related gambling practices and each other. For example, social casino spending was linked to real money video-gaming $(r=0.38)$, token wagering ( $r h o=0.34)$ and esports betting (rho=0.28). Similarly, loot box spending was strongly to both watching pre-recorded videos of loot box openings (rho=0.42) and live

Peer] reviewing PDF | (2020:01:45265:3:0:NEW 30 May 2020) 
599

600

601

602

603

604

605

606

607

608

609

610

611

612

613

614

615

616

617

618

619

620

621

622

623

624

625

626

627

628

629

630

631

632

633

634

635

636

637

638

639

640

641

642

streams of individuals opening loot boxes $(\mathrm{rho}=0.44)$. These results suggest that individuals may commonly engage in a variety of linked video-game related gambling activities in tandem, rather than a single activity (such as loot box opening or esports betting) in isolation. Indeed, it may well be the case that engagement in specific activities may contribute to engagement in others.

An interesting point may be drawn from these analyses regarding potential third factors that may be responsible for any association between loot box spending and problem gambling. During review, it was suggested that the relationship between loot box engagement and problem gambling may be a product of technological access: Individuals who are able to access loot boxes may also be able to access technology such as online betting. Further work is necessary to determine whether this is the case.

Similarly, it is interesting to note that gaming-related behaviors explained only approximately $5 \%$ of the variance in problem gambling whereas they explained over $18 \%$ of the variance in disordered gaming. One might suggest that this means that the convergence between gaming and gambling is not as important as one might expect. However, we would argue that this is not the case. In terms of magnitude, the observed relationship between problem gambling and gaming-related behaviours is stronger than relationships previously observed between problem gambling and well-known risk factors such as drug abuse and neighborhood disadvantage (Welte et al., 2006). It would be incorrect to state that the magnitude of the observed correlation makes it necessarily unimportant. In fact, we would argue that it is instead the case that the relationship between gaming-related behaviours and disordered gaming is surprisingly large, and bears further study.

\section{Limitations}

There are several potential limitations of this study that must be considered. To begin with, a sceptic may suggest that the sample that was recruited may have self-selected into the study on the basis of interest in the topic under analysis. If this were the case, one might argue that the sample used here is equivalent to a convenience sample. However, this is not the case. As noted in our method section, instructions prior to beginning the study were deliberately neutral, and only 21 participants did not complete the study after beginning it. In a sample of over 1000 participants, this represents a very small threat regarding self-selection bias.

A second point may be raised regarding the generalizability of the data uncovered here: A sceptic may suggest that the recruitment method employed (quota sampling) is unable to achieve a representative sample. Indeed, as early as 1952, the statistician Claus Moser noted that "some experts believe the quota method to be ... almost worthless. Others think that ... quota sampling can be made highly reliable, and that the heavy extra cost of random sampling does not result in a sufficient increase in accuracy to be worthwhile" (Moser, 1952). This debate has continued through the years (Cumming, 1990; Guignard et al., 2013; T. M. F. Smith, 1983). Whilst random sampling techniques may therefore represent a researcher's best chance at

Peer) reviewing PDF | (2020:01:45265:3:0:NEW 30 May 2020) 
643 reliably maximizing the representativeness of a sample, it is overly reductive to dismiss design 644 based around quota sampling as unable to represent a population under study. Indeed, such 645 designs are commonly employed to do exactly this (e.g. (Owen et al., 1998; Rubin et al., 2005; 646 Swami, 2012)).

647

648 As stated above, random sampling techniques provide the best chance for researchers to obtain 649 a maximally representative sample. This study employs purposive sampling, and interpretation 650 of prevalence from it may only be done with caution. Furthermore, within the sample itself, the 651 prevalence of disordered gambling observed here appears high (2.3\%). Estimates of this 652 magnitude have previously been observed in large-scale samples recruited via random 653 sampling methods (Economou et al., 2019; Effertz et al., 2018; Volberg et al., 2006). However, 654 in general, the incidence of problem gambling is usually estimated at less than $2 \%$ of a national 655 population (Calado \& Griffiths, 2016). Further caution must therefore be taken when using the 656 figures reported here as national prevalence estimates. These estimates should be tested via 657 658 significant subsequent work incorporating large-scale random sampling techniques.

668

669

670

Additionally, common method bias was tested for in this study using an exploratory Harman's Single Factor Test. However, as noted in (Podsakoff et al., 2003), this method is often overly incautious, and rejects the presence of bias when it really may be present. Whilst all approaches to detect common method bias have limitations, more accurate methods incorporate the measurement of latent methods factors, and the incorporation of these into formal statistical analyses (Podsakoff et al., 2003). Further research should incorporate these statistical methods. It should also focus on the measurement of factors using different and unrelated measures in order to address common method bias issues. More specifically, future research should focus on the measurement and correlation of behavioral and psychometric measures: For example, a correlation between a raw measurement of money spent on loot boxes in a video game with an individual's problem gambling severity.

An additional limitation of this study may be raised regarding the framing of our questions: As noted in our method section we endeavored to make our items as human-readable and interpretable as possible. However, it is credible that some participants may not have understood some of the questions that they were responding to. Further qualitative and scale development work is necessary to address these issues.

678

The most important limitation of this research is, however, rooted in its well-explored status as a 679 cross-sectional study: Whilst it may be able to uncover and measure correlations between

680 factors, it cannot establish precedence. Whilst gaming-related behaviours are linked to both problem gambling and disordered gaming, it is not clear which preceded or caused the other.

681 For example, loot box spending may be linked to disordered gaming because the gambling-like 682 mechanisms present in loot boxes entrap gamers, leading to the development of disordered 683 gaming. However, it is just as credible that these factors share a relationship because 684 individuals with gaming disorder tend to play games more frequently and for longer periods of 685 time, and hence are more likely to buy loot boxes. Further longitudinal studies are needed to 
686 establish Grainger causality. Significant experimental work is needed to establish the existence

687 of other forms of causality.

688

689 Conclusions

690

691

692

693

694

695

696

697

698

699

700

701

702

703

704

705

706

707

708

709

710

711

There is one clear conclusion that may be drawn from these results: The convergence of gaming and gambling is far more complex than simply the existence of loot boxes. There are a broad spectrum of inter-connected video game practices that are also similar to gambling. Many of these activities appear widespread amongst UK adults.

The widespread nature of these practices is important because all of them are significantly linked to both problem gambling and disordered gaming. In many cases these links are of a clinically significant magnitude.

The causal nature of these links is unclear. It may well be the case that engagement in practices like real-money video gaming and social casino spending are linked to problem gambling because individuals with pre-existing gambling problems are more likely to engage in these activities. However, it may also be the case that these links represent a situation in which the existence of a diverse ecosystem of gambling-like activities in video games is driving the creation of problem gambling amongst video gamers. Given the widespread nature of these practices that is suggested here, this may pose an important public health risk. Significant further work is urgently needed to further investigate these phenomena.

\section{Acknowledgements}

We would like to Acknowledge Luke Clark for his kind and thoughtful feedback on an earlier draft of this paper.

712

\section{References}

714 American Psychiatric Association. (2013). Diagnostic and statistical manual of mental disorders (DSM-5®). American Psychiatric Pub. Regular and Pathological Gambling Online Poker Players. Cyberpsychology, Behavior, and Social Networking, 16(3), 183-188. https://doi.org/10.1089/cyber.2012.0150

Brooks, G. A., \& Clark, L. (2019). Associations between loot box use, problematic gaming and 720 gambling, and gambling-related cognitions. Addictive Behaviors. 
721 Calado, F., \& Griffiths, M. D. (2016). Problem gambling worldwide: An update and systematic 722 review of empirical research (2000-2015). Journal of Behavioral Addictions, 5(4), 592-

723 613.

724

Cumming, R. G. (1990). Is probability sampling always better? A comparison of results from a 725 quota and a probability sample survey. Community Health Studies, 14(2), 132-137.

726

727

728

729

730

731

732

733

734

735

736

737

738

739

740

741

742 743

Currie, S. R., Hodgins, D. C., \& Casey, D. M. (2013). Validity of the problem gambling severity index interpretive categories. Journal of Gambling Studies, 29(2), 311-327.

Dota 2 crosses 11 million players. (2019, February 7). Mogul News. https://news.mogul.gg/dota2-11-million-players/

Dota 2-The International Battle Pass 2019. (n.d.). Dota2.Com. Retrieved September 1, 2019, from http://www.dota2.com/international/battlepass

Drummond, A., \& Sauer, J. D. (2018). Video game loot boxes are psychologically akin to gambling. Nature Human Behaviour, 530-532. https://doi.org/10.1038/s41562-018-03601

Economou, M., Souliotis, K., Malliori, M., Peppou, L. E., Kontoangelos, K., Lazaratou, H., Anagnostopoulos, D., Golna, C., Dimitriadis, G., \& Papadimitriou, G. (2019). Problem Gambling in Greece: Prevalence and Risk Factors During the Financial Crisis. Journal of Gambling Studies, 35(4), 1193-1210.

Effertz, T., Bischof, A., Rumpf, H.-J., Meyer, C., \& John, U. (2018). The effect of online gambling on gambling problems and resulting economic health costs in Germany. The European Journal of Health Economics, 19(7), 967-978.

Ferguson, C. J. (2009). An effect size primer: A guide for clinicians and researchers. Professional Psychology: Research and Practice, 40(5), 532. 
744 Ferris, J. A., \& Wynne, H. J. (2001). The Canadian problem gambling index. Canadian Centre 745 on Substance Abuse Ottawa, ON.

746 Gainsbury, S. M., Abarbanel, B., \& Blaszczynski, A. (2017). Intensity and gambling harms:

747 Exploring breadth of gambling involvement among esports bettors. Gaming Law Review, $748 \quad 21(8), 610-615$.

749 Gainsbury, S. M., Russell, A. M. T., King, D. L., Delfabbro, P., \& Hing, N. (2016). Migration 750 from social casino games to gambling: Motivations and characteristics of gamers who

751

752

753

754

755

756

757

758

759

760

761

762

763

764

765 gamble. Computers in Human Behavior, 63, 59-67. https://doi.org/10.1016/j.chb.2016.05.021

Goslin, A. (2018, December 11). The 2018 League of Legends World Finals had nearly 100 million viewers. The Rift Herald. https:/www.riftherald.com/2018/12/11/18136237/riot2018-league-of-legends-world-finals-viewers-prize-pool

Grant, J. E., Schreiber, L., Odlaug, B. L., \& Kim, S. W. (2010). Pathological Gambling and Bankruptcy. Comprehensive Psychiatry, 51(2), 115-120. https://doi.org/10.1016/j.comppsych.2009.04.002

Guignard, R., Wilquin, J.-L., Richard, J.-B., \& Beck, F. (2013). Tobacco Smoking Surveillance: Is Quota Sampling an Efficient Tool for Monitoring National Trends? A Comparison with a Random Cross-Sectional Survey. PLoS ONE, 8(10). https://doi.org/10.1371/journal.pone.0078372

Hymas, C. (2019, November 25). New laws to protect children from online harms pledged in Tory manifesto after Telegraph campaign. The Telegraph. https://www.telegraph.co.uk/politics/2019/11/25/new-laws-protect-children-onlineharms-pledged-tory-manifesto/ 
767 Kent, E. (2018, December 6). Twitch's casino section is an insidious mess - And it's right in 768 view of children. Eurogamer. https:/www.eurogamer.net/articles/2018-12-06-twitchs769 casino-section-is-an-insidious-mess-and-its-right-in-view-of-children

770 Kent, E. (2019, March 5). Game companies should share data on loot boxes, academics tell 771 Parliament. Eurogamer. https://www.eurogamer.net/articles/2019-03-05-game772 companies-should-share-data-on-loot-boxes-say-academics

773 Kim, H. S., Wohl, M. J. A., Salmon, M. M., Gupta, R., \& Derevensky, J. (2015). Do Social 774 Casino Gamers Migrate to Online Gambling? An Assessment of Migration Rate and 775 Potential Predictors. Journal of Gambling Studies, 31(4), 1819-1831.

776 https://doi.org/10.1007/s10899-014-9511-0

777 King, D. L., \& Delfabbro, P. H. (2018). Video Game Monetization (e.g., 'Loot Boxes'): A 778 Blueprint for Practical Social Responsibility Measures. International Journal of Mental 779 Health and Addiction. https://doi.org/10.1007/s11469-018-0009-3

780

781

782

783

784

785

786

787

788

789

King, D. L., \& Delfabbro, P. H. (2020). The convergence of gambling and monetised gaming activities. Current Opinion in Behavioral Sciences, 31, 32-36.

Klepek, P. (2019, August 15). What Happens to a Streamer Known for Opening Loot Boxes After They’re Gone? Vice. https://www.vice.com/en_us/article/7x598q/what-happens-toa-streamer-known-for-opening-loot-boxes-after-theyre-gone

Lemmens, J. S., Valkenburg, P. M., \& Gentile, D. A. (2015). The Internet gaming disorder scale. Psychological Assessment, 27(2), 567.

Li, W., Mills, D., \& Nower, L. (2019). The relationship of loot box purchases to problem video gaming and problem gambling. Addictive Behaviors, 97, 27-34. https://doi.org/10.1016/j.addbeh.2019.05.016 
790 M. Gainsbury, S., Hing, N., Delfabbro, P. H., \& King, D. L. (2014). A taxonomy of gambling 791 and casino games via social media and online technologies. International Gambling $792 \quad$ Studies, 14(2), 196-213.

793 Macey, J., \& Hamari, J. (2018). Investigating relationships between video gaming, spectating 794

795 esports, and gambling. Computers in Human Behavior, 80, 344-353. https://doi.org/10.1016/j.chb.2017.11.027

796

797

798

799

800

801

802

803

804

805

806

807

808

809

810

811

812

MacKenzie, S. B., \& Podsakoff, P. M. (2012). Common method bias in marketing: Causes, mechanisms, and procedural remedies. Journal of Retailing, 88(4), 542-555.

Moser, C. A. (1952). Quota Sampling. Journal of the Royal Statistical Society. Series A (General), 115(3), 411-423. JSTOR. https://doi.org/10.2307/2980740

Orford, J., Wardle, H., Griffiths, M., Sproston, K., \& Erens, B. (2010). PGSI and DSM-IV in the 2007 British Gambling Prevalence Survey: Reliability, item response, factor structure and inter-scale agreement. International Gambling Studies, 10(1), 31-44. https://doi.org/10.1080/14459790903567132

Owen, L., McNeill, A., \& Callum, C. (1998). Trends in smoking during pregnancy in England, 1992-7: Quota sampling surveys. BMJ : British Medical Journal, 317(7160), 728-730.

Petry, N. M., \& Kiluk, B. D. (2002). Suicidal ideation and suicide attempts in treatment-seeking pathological gamblers. The Journal of Nervous and Mental Disease, 190(7), 462.

Podsakoff, P. M., MacKenzie, S. B., Lee, J.-Y., \& Podsakoff, N. P. (2003). Common method biases in behavioral research: A critical review of the literature and recommended remedies. Journal of Applied Psychology, 88(5), 879.

Raylu, N., \& Oei, T. P. (2004). Role of culture in gambling and problem gambling. Clinical Psychology Review, 23(8), 1087-1114. 
813 Rubin, G. J., Brewin, C. R., Greenberg, N., Simpson, J., \& Wessely, S. (2005). Psychological

814 and behavioural reactions to the bombings in London on 7 July 2005: Cross sectional

815 survey of a representative sample of Londoners. Bmj, 331(7517), 606.

816 Smith, C. (2019, January 20). Esports Betting Market Set to Explode to a Total Value of \$12.9B

817 by 2020. Casino.Org. https://www.casino.org/news/esports-betting-market-set-to-

818 explode-to-a-total-value-of-12-9b-by-2020

819 Smith, T. M. F. (1983). On the Validity of Inferences from Non-Random Samples. Journal of the

820 Royal Statistical Society: Series A (General), 146(4), 394-403.

821 https://doi.org/10.2307/2981454

822 Swami, V. (2012). Mental health literacy of depression: Gender differences and attitudinal 823 antecedents in a representative British sample. PloS One, 7(11).

824 Turner, N. E., Elton-Marshall, T., Shi, J., Wiebe, J., Boak, A., van der Maas, M., \& Mann, R. E.

825 (2018). Cross Validation of the Gambling Problem Severity Subscale of the Canadian

826 Adolescent Gambling Index (CAGI/GPSS) on a Sample of Ontario High School

827 Students. Journal of Gambling Studies, 34(2), 521-537. https://doi.org/10.1007/s10899-

$828 \quad 017-9731-1$

829 Veselka, L., Wijesingha, R., Leatherdale, S. T., Turner, N. E., \& Elton-Marshall, T. (2018).

830 Factors associated with social casino gaming among adolescents across game types. BMC $831 \quad$ Public Health, 18(1), 1167.

832 Volberg, R. A., Nysse-Carris, K. L., \& Gerstein, D. R. (2006). 2006 California problem

833 gambling prevalence survey. National Opinion Research Center (NORC). 
834 Wardle, H., Moody, A., Spence, S., Orford, J., Volberg, R., Jotangia, D., Griffiths, M., Hussey, 835 D., \& Dobbie, F. (2011). British gambling prevalence survey 2010. The Stationery $836 \quad$ Office.

837 Welte, J. W., Wieczorek, W. F., Barnes, G. M., \& Tidwell, M.-C. O. (2006). Multiple risk 838 factors for frequent and problem gambling: Individual, social, and ecological. Journal of Applied Social Psychology, 36(6), 1548-1568.

840

Zendle, D. (2019a). Only problem gamblers spend less money when loot boxes are removed from

841 a game: A before and after study of Heroes of the Storm.

842 https://doi.org/10.31234/osf.io/a3kp2

Zendle, D. (2019b). Is real-money mobile gaming a form of gambling or video gaming?

844 [Preprint]. PsyArXiv. https://doi.org/10.31234/osf.io/9wp5h

845

846

Zendle, D., \& Cairns, P. (2018). Video game loot boxes are linked to problem gambling: Results of a large-scale survey. PLoS One, 13(11), e0206767.

847 Zendle, D., \& Cairns, P. (2019). Loot boxes are again linked to problem gambling: Results of a 848 replication study. PLoS One, 14(3), e0213194.

849 https://doi.org/10.1371/journal.pone.0213194

850

851

852

853 854 855
Zendle, D., McCall, C., Barnett, H., \& Cairns, P. (2018). Paying for loot boxes is linked to problem gambling, regardless of specific features like cash-out and pay-to-win: A preregistered investigation. https://doi.org/10.31234/osf.io/6e 74k

Zendle, D., Meyer, R., \& Over, H. (2019). Adolescents and loot boxes: Links with problem gambling and motivations for purchase. Royal Society Open Science, 6(6), 190049. https://doi.org/10.1098/rsos.190049 
856 Zendle, D., Meyer, R., Waters, S., \& Cairns, P. (2020). The prevalence of loot boxes in mobile 857 and desktop games. Addiction.

858 https://onlinelibrary.wiley.com/doi/abs/10.1111/add.14973?casa_token=JnwIbkbkPxQA 859 AAAA:1ZfA6Xz198biEsPPIGmW1JA0r31YtoG4GKXoSb9qghAnX860 S0UPa4Js6npuwBP5T1mCmxaRafalt71Q

861 


\section{Table $\mathbf{1}$ (on next page)}

Prevalence estimates for both traditional gambling and gaming-related gambling behaviours, with $95 \%$ confidence interval calculated according to the adjusted Wald procedure. 


\begin{tabular}{|c|c|c|c|c|}
\hline $\begin{array}{l}\text { Type of } \\
\text { behaviour }\end{array}$ & Behaviour & $\begin{array}{l}\text { Proportion engaging in } \\
\text { activity at least once in } \\
\text { the past } 12 \text { months }\end{array}$ & LLCl & ULCI \\
\hline \multirow{10}{*}{$\begin{array}{l}\text { Gambling- } \\
\text { like video } \\
\text { game } \\
\text { practices }\end{array}$} & Any form of gambling-like video game practice & $18.5 \%$ & $16.3 \%$ & $20.9 \%$ \\
\hline & Esports betting & $2.9 \%$ & $2.0 \%$ & $4.1 \%$ \\
\hline & Loot box spending & $7.8 \%$ & $6.4 \%$ & $9.6 \%$ \\
\hline & Social casino spending & $3.7 \%$ & $2.7 \%$ & $5.0 \%$ \\
\hline & Real-money video gaming & $1.7 \%$ & $1.1 \%$ & $2.7 \%$ \\
\hline & Token wagering & $2.2 \%$ & $1.4 \%$ & $3.3 \%$ \\
\hline & Watching loot box openings online (live) & $4.8 \%$ & $3.6 \%$ & $6.2 \%$ \\
\hline & Watching loot box openings online (pre-recorded) & $6.6 \%$ & $5.3 \%$ & $8.3 \%$ \\
\hline & Watching gambling online (live) & $4.1 \%$ & $3.1 \%$ & $5.5 \%$ \\
\hline & Watching gambling online (pre-recorded) & $4.2 \%$ & $3.1 \%$ & $5.6 \%$ \\
\hline \multirow{12}{*}{$\begin{array}{l}\text { Traditional } \\
\text { forms of } \\
\text { gambling }\end{array}$} & Any form of traditional gambling & $71.3 \%$ & $68.5 \%$ & $73.9 \%$ \\
\hline & Purchasing lottery tickets & $48.9 \%$ & $45.9 \%$ & $51.9 \%$ \\
\hline & Purchasing instant win / scratch cards & $34.7 \%$ & $32.0 \%$ & $37.6 \%$ \\
\hline & Betting on sports events (excluding esports) & $27.6 \%$ & $25.0 \%$ & $30.4 \%$ \\
\hline & Betting on horse or dog racing & $21.7 \%$ & $19.3 \%$ & $24.3 \%$ \\
\hline & Playing bingo for money in person & $8.2 \%$ & $6.7 \%$ & $10.0 \%$ \\
\hline & Playing bingo for money online & $11.1 \%$ & $9.4 \%$ & $13.2 \%$ \\
\hline & $\begin{array}{l}\text { Playing games of skill for money against other } \\
\text { individuals }\end{array}$ & $6.8 \%$ & $5.4 \%$ & $8.5 \%$ \\
\hline & Playing slot machines in person & $13.3 \%$ & $11.4 \%$ & $15.4 \%$ \\
\hline & Playing slot machines online & $14.4 \%$ & $12.4 \%$ & $16.6 \%$ \\
\hline & Playing casino table games in a casino & $7.4 \%$ & $5.9 \%$ & $9.1 \%$ \\
\hline & Playing casino table games online & $10.4 \%$ & $8.7 \%$ & $12.4 \%$ \\
\hline
\end{tabular}

1

2 Table 1: Prevalence estimates for both traditional gambling and gaming-related gambling behaviours,

3 with 95\% confidence interval calculated according to the adjusted Wald procedure. LLCI stands for the

4 lower limit of the confidence interval; ULCI stands for the upper limit of the confidence interval. 


\section{Table 2 (on next page)}

The relationship of gambling-like video game practices with both disordered gaming and problem gambling.

Relationships that are significant at the $p<0.05$ level are marked *; $p<0.01$ are marked **; $p<0.001$ are marked $* * *$. 


\begin{tabular}{|c|c|c|}
\hline $\begin{array}{c}\text { Gambling-like video game } \\
\text { practice }\end{array}$ & $\begin{array}{c}\text { Relationship with problem } \\
\text { gambling (Spearman's rho) }\end{array}$ & $\begin{array}{c}\text { Relationship with disordered } \\
\text { gaming (Spearman's rho) }\end{array}$ \\
\hline $\begin{array}{c}\text { Any form of gambling-like video } \\
\text { game practice }\end{array}$ & $0.23^{* * *}$ & $0.43^{* * *}$ \\
\hline Esports betting & $0.21^{* * *}$ & $0.14^{* * *}$ \\
\hline Loot box spending & $0.14^{* * *}$ & $0.41^{* * *}$ \\
\hline Social casino spending & $0.21^{* * *}$ & $0.17^{* * *}$ \\
\hline Real-money video gaming & $0.15^{* * *}$ & $0.18^{* * *}$ \\
\hline Token wagering & $0.12^{* * *}$ & $0.18^{* * *}$ \\
\hline $\begin{array}{c}\text { Watching loot box openings } \\
\text { online (live) }\end{array}$ & $0.14^{* * *}$ & $0.32^{* * *}$ \\
\hline $\begin{array}{c}\text { Watching loot box openings } \\
\text { online (pre-recorded) }\end{array}$ & $0.13^{* * *}$ & $0.35^{* * *}$ \\
\hline Watching gambling online (live) & $0.20^{* * *}$ & $0.30^{* * *}$ \\
\hline $\begin{array}{c}\text { Watching gambling online (pre- } \\
\text { recorded) }\end{array}$ & $0.17^{* * *}$ & $0.25^{* * *}$ \\
\hline
\end{tabular}

2

3 Table 2: The relationship of gambling-like video game practices with both disordered gaming and

4 problem gambling. Relationships that are significant at the $p<0.05$ level are marked $* ; p<0.01$ are

5 marked $* * ; p<0.001$ are marked $* * *$. 


\section{Table 3 (on next page)}

Spearman's rho correlation coefficient between engagement in traditional gambling activities and engagement in gaming-related gambling activities.

Relationships that are significant at the $p<0.05$ level are marked *; $p<0.01$ are marked **; $p<0.001$ are marked $* * *$. 


\begin{tabular}{|c|c|c|c|c|c|c|c|c|c|}
\hline & 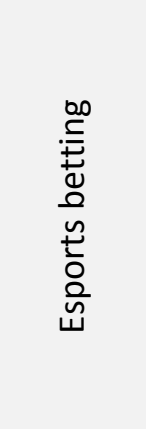 & 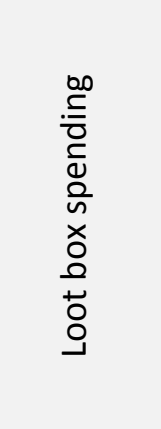 & 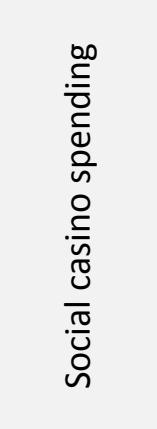 & 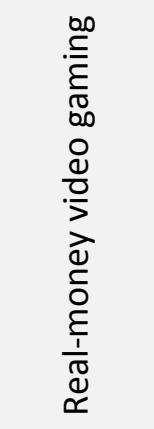 & 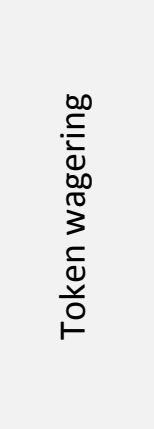 & 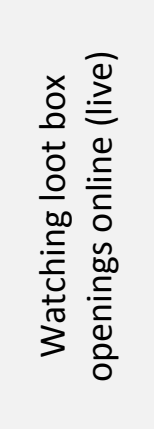 & 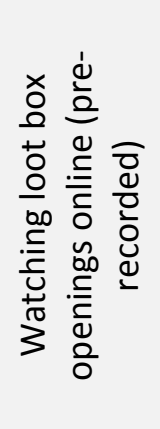 & 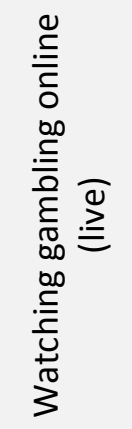 & 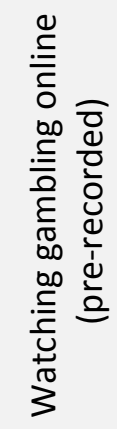 \\
\hline Any form of traditional gambling & $0.09 * *$ & 0.04 & $0.13 * * *$ & $0.07 *$ & 0.03 & $<0.01$ & $<0.01$ & 0.03 & 0.07 \\
\hline Purchasing lottery tickets & -0.02 & -0.05 & $0.07 *$ & 0.05 & -0.03 & $-0.08 * *$ & $-0.09 * *$ & -0.04 & -0.03 \\
\hline Purchasing instant win / scratch cards & $0.08^{* *}$ & $0.07^{*}$ & $0.15^{* * *}$ & $0.13^{* * *}$ & $0.06^{*}$ & 0.04 & 0.01 & $0.06^{*}$ & 0.05 \\
\hline $\begin{array}{c}\text { Betting on sports events (excluding } \\
\text { esports) }\end{array}$ & $0.18 * * *$ & $0.08^{* *}$ & $0.17 * * *$ & $0.12 * * *$ & $0.07^{*}$ & 0.03 & 0.02 & 0.05 & $0.08^{* *}$ \\
\hline Betting on horse or dog racing & $0.07^{*}$ & 0.01 & $0.09 * *$ & $0.06 *$ & 0.04 & -0.04 & -0.07 & 0.05 & 0.02 \\
\hline Playing bingo in person for money & $0.08^{* *}$ & 0.03 & $0.12^{* * *}$ & $0.13^{* * *}$ & $0.06 *$ & $<0$ & 0.01 & $0.07^{*}$ & $0.08^{* *}$ \\
\hline Playing bingo online for money & $0.14^{* * *}$ & $0.07^{*}$ & $0.19 * * *$ & $0.21 * * *$ & $0.08 * *$ & 0.05 & $0.06 *$ & $0.09 * *$ & $0.07^{*}$ \\
\hline Playing games of skill for money & $0.22 * * *$ & $0.27 * * *$ & $0.22 * * *$ & $0.17 * * *$ & $0.16^{* * *}$ & $0.19 * * *$ & $0.18^{* * *}$ & $\begin{array}{c}0.28^{* *} \\
*\end{array}$ & $\begin{array}{c}0.24^{* *} \\
*\end{array}$ \\
\hline Playing slot machines in person & $0.08^{* *}$ & $0.12 * * *$ & $0.17^{* * *}$ & $0.11 * * *$ & $0.06 *$ & 0.05 & $0.07^{*}$ & $\begin{array}{c}0.14^{* *} \\
*\end{array}$ & $\begin{array}{c}0.12^{* *} \\
*\end{array}$ \\
\hline Playing slot machines online & $0.23 * * *$ & $0.16 * * *$ & $0.26 * * *$ & $0.19 * * *$ & $0.09 * *$ & $0.12 * * *$ & $0.13^{* * *}$ & $\begin{array}{c}0.14^{* *} \\
*\end{array}$ & $\begin{array}{c}0.12^{* *} \\
*\end{array}$ \\
\hline Playing casino table games in a casino & $0.13^{* * *}$ & $0.13^{* * *}$ & $0.21 * * *$ & $0.09 * *$ & $0.06^{*}$ & 0.05 & 0.04 & $\begin{array}{c}0.12^{* *} \\
*\end{array}$ & $\begin{array}{l}0.10^{* *} \\
*\end{array}$ \\
\hline Playing casino table games online & $0.24 * * *$ & $0.21 * * *$ & $0.28 * * *$ & $0.22 * * *$ & $0.18^{* * *}$ & $0.12^{* * *}$ & $0.13^{* * *}$ & $\begin{array}{l}0.20^{* *} \\
*\end{array}$ & $\begin{array}{c}0.20 * * \\
*\end{array}$ \\
\hline
\end{tabular}


3 Table 3: Spearman's rho correlation coefficient between engagement in traditional gambling activities and engagement in gaming-related 4 gambling activities. Relationships that are significant at the $p<0.05$ level are marked $* ; p<0.01$ are marked $* * ; p<0.001$ are marked $* * *$. 


\section{Table 4 (on next page)}

Spearman's rho correlation coefficient between engagement in each gambling-like video game practice.

Relationships that are significant at the $p<0.05$ level are marked *; $p<0.01$ are marked **; $p<0.001$ are marked $* * *$. 


\begin{tabular}{|c|c|c|c|c|c|c|c|}
\hline & 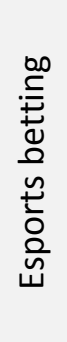 & 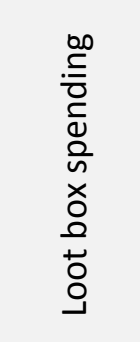 & $\begin{array}{l}\frac{\pi}{0} \\
\frac{0}{0} \\
\frac{0}{\sqrt{n}} \\
\frac{0}{0} \\
\frac{\pi}{4} \\
\text { D }\end{array}$ & 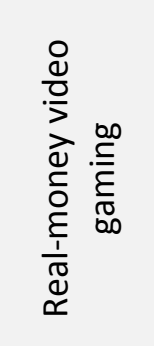 & 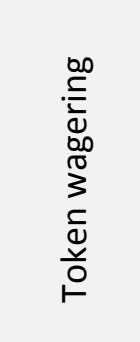 & 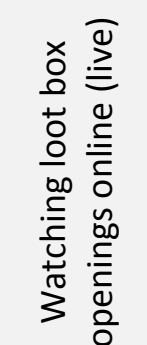 & 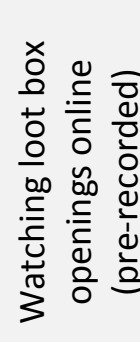 \\
\hline Esports betting & 1 & $0.16 * * *$ & $0.28 * * *$ & $0.25^{* * *}$ & $0.31 * * *$ & $0.25 * * *$ & $0.21 * * *$ \\
\hline Loot box spending & & 1 & $0.2 * * *$ & $0.2^{* * *}$ & $0.24 * * *$ & $0.44 * * *$ & $0.42 * * *$ \\
\hline Social casino spending & & & 1 & $0.38 * * *$ & $0.34 * * *$ & $0.25 * * *$ & $0.21 * * *$ \\
\hline Real-money video gaming & & & & 1 & $0.4^{* * *}$ & $0.23 * * *$ & $0.2 * * *$ \\
\hline Token wagering & & & & & 1 & $0.35 * * *$ & $0.32 * * *$ \\
\hline $\begin{array}{l}\text { Watching loot box } \\
\text { openings online (live) }\end{array}$ & & & & & & 1 & $0.69 * * *$ \\
\hline $\begin{array}{l}\text { Watching loot box } \\
\text { openings online } \\
\text { (pre-recorded) }\end{array}$ & & & & & & & 1 \\
\hline
\end{tabular}

2 Table 4: Spearman's rho correlation coefficient between engagement in each gambling-like video

3 game practice. Relationships that are significant at the $p<0.05$ level are marked $* ; p<0.01$ are marked 


\section{Table 5 (on next page)}

Table 5: Ordinal logistic regression of the influence of gaming-related behaviours, gender, and age on problem gambling. Odds Ratios are reported as a measure of effect size.

These represent the influence of a single point difference in a factor on the likelihood that an individual is in a group other than non-problem gamblers.

Behaviours are measured on a frequency scale of $0-3$. Gender is coded as $0-1$, with 0 being female. Predictors that are significant at the $p<0.05$ level are marked *; $p<0.01$ are marked $* * ; p<0.001$ are marked ***. 


\begin{tabular}{|c|c|c|c|c|c|c|c|c|c|c|c|c|}
\hline \multicolumn{5}{|c|}{ Gaming-related behavior } & \multicolumn{4}{|c|}{ Gender } & \multicolumn{4}{|c|}{ Age } \\
\hline Behaviour name & $\beta$ & SE & $\mathrm{t}$ & OR & $\beta$ & SE & $\mathrm{t}$ & OR & $\beta$ & SE & $\mathrm{t}$ & OR \\
\hline Esports betting & 0.55 & 0.09 & $5.87 * * *$ & 1.73 & -0.01 & $<0.01$ & $-2.76 * *$ & 0.99 & 0.41 & 0.14 & $2.92 * *$ & 1.50 \\
\hline Loot box spending & 0.31 & 0.09 & $3.37 * * *$ & 1.36 & -0.01 & $<0.01$ & $-2.60 * *$ & 0.99 & 0.38 & 0.14 & $2.74 * *$ & 1.47 \\
\hline Social casino play & 0.62 & 0.10 & $6.31 * * *$ & 1.87 & -0.01 & $<0.01$ & $-2.78 * *$ & 0.99 & 0.40 & 0.14 & $2.85^{* *}$ & 1.49 \\
\hline $\begin{array}{c}\text { Real-money video } \\
\text { gaming }\end{array}$ & 0.58 & 0.15 & $3.77 * * *$ & 1.79 & -0.01 & $<0.01$ & $-3.13 * *$ & 0.99 & 0.42 & 0.14 & $3.01 * *$ & 1.52 \\
\hline Token wagering & 0.46 & 0.13 & $3.54 * * *$ & 1.59 & -0.01 & $<0.01$ & $-2.97 * *$ & 0.99 & 0.41 & 0.14 & $2.98 * *$ & 1.51 \\
\hline $\begin{array}{l}\text { Watching loot boxes } \\
\text { (live) }\end{array}$ & 0.43 & 0.10 & $4.37 * * *$ & 1.54 & -0.01 & $<0.01$ & $-2.32^{*}$ & 0.99 & 0.37 & 0.14 & $2.61 * *$ & 1.44 \\
\hline $\begin{array}{c}\text { Watching loot boxes } \\
\text { (pre-recorded) }\end{array}$ & 0.39 & 0.09 & $4.14 * * *$ & 1.48 & -0.01 & $<0.01$ & $-2.02^{*}$ & 0.99 & 0.36 & 0.14 & $2.56^{*}$ & 1.43 \\
\hline $\begin{array}{c}\text { Watching gambling } \\
\text { online (live) }\end{array}$ & 0.47 & 0.09 & $5.24 * * *$ & 1.60 & -0.01 & $<0.01$ & $-2.49 *$ & 0.99 & 0.36 & 0.14 & $2.61^{* *}$ & 1.44 \\
\hline $\begin{array}{l}\text { Watching gambling } \\
\text { online (pre-recorded) }\end{array}$ & 0.37 & 0.08 & $4.42 * * *$ & 1.45 & -0.01 & $<0.01$ & $-2.77^{* *}$ & 0.99 & 0.35 & 0.14 & $2.46^{*}$ & 1.41 \\
\hline
\end{tabular}

2 Table 5: Ordinal logistic regression of the influence of gaming-related behaviours, gender, and age on problem gambling. Odds Ratios are reported as a measure of effect size. These represent the influence of a single point difference in a factor on the likelihood that an individual is in a group other than non-problem gamblers. Behaviours are measured on a frequency scale of 0-3. Gender is coded as $0-1$, with 0 being female. Predictors that are significant at the $p<0.05$ level are marked $* ;<0.01$ are marked $* * ; p<0.001$ are marked $* * *$. 


\section{Table 6(on next page)}

Table 6: Logistic regression of the association between gaming-related behaviours, gender, and age on disordered gaming. Odds Ratios are reported as a measure of effect size.

These represent the influence of a single point difference in a factor on the likelihood that an individual is classified as a disordered gamer. Behaviours are measured on a frequency scale of 0-3. Gender is coded as 0-1, with 0 being female. Predictors that are significant at the $p<0.05$ level are marked $* ;<0.01$ are marked $* * ; p<0.001$ are marked $* * *$. 


\begin{tabular}{|c|c|c|c|c|c|c|c|c|c|c|c|c|}
\hline \multicolumn{5}{|c|}{ Gaming-related behaviour } & \multicolumn{4}{|c|}{ Gender } & \multicolumn{4}{|c|}{ Age } \\
\hline Behaviour name & $\beta$ & SE & $z$ & OR & $\beta$ & SE & z & OR & $\beta$ & SE & z & OR \\
\hline Esports betting & 0.31 & 0.11 & $2.73 * *$ & 1.36 & 0.51 & 0.25 & $2.08 *$ & 1.67 & -0.04 & 0.01 & $-4.64 * * *$ & 0.96 \\
\hline Loot box spending & 0.69 & 0.12 & $5.83^{* * *}$ & 2.00 & 0.32 & 0.25 & 1.27 & 1.38 & -0.03 & 0.01 & $-3.51 * * *$ & 0.97 \\
\hline Social casino play & 0.39 & 0.12 & $3.33^{* * *}$ & 1.47 & 0.50 & 0.25 & $2.03^{*}$ & 1.65 & -0.04 & 0.01 & $-4.70 * * *$ & 0.96 \\
\hline Real-money video gaming & 0.41 & 0.18 & $2.23 *$ & 1.50 & 0.51 & 0.24 & $2.09 *$ & 1.67 & -0.04 & 0.01 & $-4.72 * * *$ & 0.96 \\
\hline Token wagering & 0.66 & 0.16 & $4.21 * * *$ & 1.94 & 0.49 & 0.25 & 1.96 & 1.63 & -0.04 & 0.01 & $-4.23 * * *$ & 0.96 \\
\hline Watching loot boxes (live) & 0.77 & 0.14 & $5.53^{* * *}$ & 2.16 & 0.29 & 0.26 & 1.12 & 1.33 & -0.03 & 0.01 & $-3.06 * *$ & 0.97 \\
\hline $\begin{array}{l}\text { Watching loot boxes (pre- } \\
\text { recorded) }\end{array}$ & 0.64 & 0.13 & $5.08 * * *$ & 1.90 & 0.30 & 0.25 & 1.19 & 1.35 & -0.03 & 0.01 & $-2.88 * *$ & 0.97 \\
\hline $\begin{array}{l}\text { Watching gambling online } \\
\text { (live) }\end{array}$ & 0.53 & 0.11 & $4.98 * * *$ & 1.71 & 0.42 & 0.25 & 1.66 & 1.52 & -0.03 & 0.01 & $-3.90 * * *$ & 0.97 \\
\hline $\begin{array}{l}\text { Watching gambling online } \\
\text { (pre-recorded) }\end{array}$ & 0.44 & 0.11 & $4.04 * * *$ & 1.55 & 0.34 & 0.25 & 1.33 & 1.40 & -0.04 & 0.01 & $-4.23 * * *$ & 0.96 \\
\hline
\end{tabular}

1

2 Table 6: Logistic regression of the influence of gaming-related behaviours, gender, and age on disordered gaming. Odds Ratios are 3 reported as a measure of effect size. These represent the influence of a single point difference in a factor on the likelihood that an 4 individual is classified as a disordered gamer. Behaviours are measured on a frequency scale of 0-3. Gender is coded as 0-1, with 0 5 being female. Predictors that are significant at the $p<0.05$ level are marked $* ; p<0.01$ are marked $* * ; p<0.001$ are marked $* * *$. 
